\title{
Catheter Ablation of Non-inducible Atrial Tachycardia After Surgical Repair of Heart
}

Disease

Takeshi Tomita MD, Kazunori Aizawa MD, Takahiro Takeuchi, MD, Kentaro Shimada, MD, Ayako Okada MD, Megumi Koshikawa MD, Hiroki Kasai MD, Atsushi Izawa MD, Yusuke Miyashita, MD, Setsuo Kumazaki MD, Jun Koyama MD, Uichi Ikeda $\mathrm{MD}$,

Department of Cardiovascular Medicine, Shinshu University School of Medicine 3-1-1 Asahi Matsumoto Nagano, Japan

Address for correspondence to:

Takeshi Tomita MD,

Department of Cardiovascular Medicine, Shinshu University School of Medicine 3-1-1 Asahi Matsumoto Nagano, Japan

E-mail; ttomita@shinshu-u.ac.jp 


\begin{abstract}
We present a patient with non-inducible atrial tachycardia (AT) after atriotomy for surgical repair of heart disease who underwent ablation successfully. Using a 3D mapping system, we presumed the atriotomy site on the lateral right atrial wall by searching for linear double potentials (DP) during sinus/paced rhythm from the coronary sinus, but it was evaluated incompletely. We could verify the edges of the atriotomy scar precisely by pacing from close to the linear DP lesion and the opposite site. After ablation between the presumed atriotomy scar and the inferior vena cava and cavotricuspid isthmus, no AT recurred without anti-arrhythmic drugs.
\end{abstract}

Key Words: atrial tachycardia; catheter ablation; incision; atriotomy 


\section{Introduction}

Atrial tachycardia (AT) is observed occasionally in patients after atriotomy for surgical repair of heart disease [1][2]. Sustained AT generally results from macroreentry around the atriotomy scar and cavotricuspid isthmus (CTI)-dependent atrial flutter (AFL). In such cases, we can identify the reentrant circuit using a 3-dimensional electroanatomical mapping system (3D mapping) [3][4]. In a subset of cases, however, AT/AFLs are short in duration and/or noninducible, despite pharmacological provocation prior to catheter ablation. We report that the usefulness of multiple site pacing using a 3D mapping system to identify the atriotomy scar precisely in such patients.

\section{Case report}

An 82-year-old man had been suffering from paroxysmal AT (Fig. 1) since he underwent right atriotomy of the lateral wall for mitral valve replacement with tricuspid annuloplasty 4 years ago. The 12-lead electrocardiogram showed a negative $\mathrm{P}$ wave (sawtooth-like waveform) in leads II, III, aVf and positive $\mathrm{P}$ wave in leads $\mathrm{I}$ and $\mathrm{aV}$. We guessed that the mechanism of the paroxysmal AT was combined clockwise incisional AT and common-type AFL with 2:1 AV conduction. He received several anti-arrhythmic drugs, but all medications failed to eliminate AT. He was transferred to our hospital emergently because of frequent AT episodes and congestive heart failure. 
We planned catheter ablation to prevent AT after the improvement of heart failure. Anti-arrhythmic medications were discontinued 7 days before the electrophysiological study. Electrocardiograms prior to ablation displayed sinus rhythm. Although we attempted to induce clinical AT by atrial stimulation with beta-agonist infusion, no atrial arrhythmias could be induced. Therefore, we planned to obtain a voltage map for finding the channels of AT and create a conduction block line between the channels and/or the atriotomy scar and the inferior vena cava (IVC) to prevent incisional reentrant AT. Using the CARTO XP system (Biosene-Webster, Diamond Bar, CA, USA), we obtained a right atrial activation map to identify the atriotomy scar by searching for linear double potentials (DPs) [3]. A DP was defined by the presence on the atrial electrogram of 2 discrete deflections per beat, separated by an isoelectric baseline [5]. The local atrial electrogram for constructing an activation map was determined by the following: (1) rapid atrial deflection, (2) consistency of the nearby atrial electrogram, (3) relation between the pacing site and the linear DP region. Areas with no atrial potential distinguishable from noise $(<0.05 \mathrm{mV})$ were considered dense scars and were displayed in gray.

During sinus rhythm, a low voltage area was noted on lower lateral wall. DPs were not noted clearly on the lateral wall. Following coronary sinus pacing, the scar area in 
low voltage area was noted on narrow linear region of mid-lateral wall of RA (Fig. 2E).

Several split potentials beside the scar area were noted on the lateral wall (Fig. 2B). During pacing from a region close to a split potential, a linear DP region was recognized (Fig. 3, 4; B, and D). However, the electrocardiogram at the upper boundary of the linear lesion near the pacing site showed a single potential (Fig. 3, 4; A) or indiscrete DP (Fig. 3, 4; C). Low voltage and fragmented potentials were noted between the lower boundary of the DP lesion and the IVC (Fig. 3, 4; E). Pacing from the opposite site revealed another discrete DP that was not seen in panel A and C of Fig. 4 (Fig. $5 \mathrm{C}$ and D). The intervals between the first and the second deflection of the DP around the mid portion of the linear DP region (Fig. 5 A; 72ms) were relatively long compared with those around the edges, gradually shortening toward the edges (Fig. 5 C; 38ms). Eventually, a single potential was recognized after the disappearance of DPs or split potentials. Additional multiple site pacing allowed clarification of the edges of the DP region, indicating the location of the atriotomy scar (Fig. 5). There were no channels associated with AT in other RA area.

Radiofrequency application using a Navistar DS 8-mm tip catheter (Biosense Webster, USA) was delivered between the lower portion of the presumed incisional scar and the IVC (Fig. 6, red tags). After completion of ablation, bi-directional conduction 
blockade across the linear lesion was verified by pacing close to the ablation line. In addition, ablation of the CTI was performed until a complete bi-directional conduction block was achieved (Fig. 6, red tags). Finally, programmed stimulation was repeated to confirm the non-inducibility of AT. The follow-up period was 14 months after ablation. 12-lead electrocardiogram every month revealed no ATs without the use of anti-arrhythmic drugs.

\section{Discussion}

Electrophysiological studies in patients with AT after surgical repair are generally performed for stable AT, allowing easy identification of the reentrant circuit [1][2]. In this patient, however, AT could not be induced despite any provocation methods. A favorable condition of hemodynamics after the appropriate therapy for heart failure might result in non-inducibility. Love et al. demonstrated that the atriotomy scar can be identified by electroanatomical mapping in sinus/atrially paced rhythm from the epicardium, esophagus and coronary sinus [5]. However, we could not record DPs clearly during sinus rhythm and paced rhythm from the coronary sinus. When the activation wave-front comes toward the atriotomy scar vertically, the DPs become discrete in comparison with those seen when the wave-front is running parallel to the scar. The activation wave-front from the sinus node and coronary sinus generally runs 
parallel to the atriotomy scar. Pacing from beside the edge of the atriotomy scar would also result in indiscrete DPs. Therefore, pacing from several different atrial sites, especially those close to the mid portion of the presumed atriotomy scar, is required to identify discrete DPs in the lateral wall and verify the superior and inferior borders of the lesion.

In previous reports, both CTI and incision related AT/AFL have been shown to frequently coexist after atriotomy for valve repair [6]. Therefore, the composite ablation of the atriotomy scar-the IVC and the CTI would be necessary and effective for the prevention of AT/AFLs after surgical atriotomy. We could not verify the effectiveness of ablation. However, no recurrence of AT and AFLs was recognized without anti-arrhythmic drugs. Therefore, we believe that the catheter ablation eliminated the AT in this case.

Valve surgery can be performed in octogenarian patients with acceptable mortality and good long-term results;early referral for surgery is important to achieve a better postoperative outcome [7]. In addition, as in this case, atrial tachycardias related atriotomy should also be eliminated to maintain a good quality of life.

In conclusion, searching for linear DPs during pacing from several atiral sites with using a 3D mapping system was useful for identifying the edges of the atriotomy scar in 
patients with non-inducible AT. The composite ablation of the presumed atriotomy scar-the IVC and the CTI could eliminate AT. 


\section{References}

[1] Kalman JM, VanHare GF, Olgin JE, Saxon LA, Stark SI, Lesh MD (1996)

Ablation of 'incisional' reentrant atrial tachycardia complicating surgery for congenital heart disease. Use of entrainment to define a critical isthmus of conduction. Circulation 93:502-12

[2] Baker BM, Lindsay BD, Bromberg BI, Frazier DW, Cain ME, Smith JM (1996) Catheter ablation of clinical intraatrial reentrant tachycardias resulting from previous atrial surgery: localizing and transecting the critical isthmus. J Am Coll Cardiol 28:411-7

[3] Nakagawa H, Shah N, Matsudaira K, Overholt E, Chandrasekaran K, Beckman KJ, Spector P, Calame JD, Rao A, Hasdemir C, Otomo K, Wang Z, Lazzara R, Jackman WM (2001) Characterization of reentrant circuit in macroreentrant right atrial tachycardia after surgical repair of congenital heart disease: isolated channels between scars allow "focal" ablation. Circulation 103:699-709

[4] Magnin-Poull I, De Chillou C, Miljoen H, Andronache M, Aliot E (2005) Mechanisms of right atrial tachycardia occurring late after surgical closure of atrial septal defects. J Cardiovasc Electrophysiol 16:681-7

[5] Love BA, Collins KK, Walsh EP, Triedman JK (2001) Electroanatomic 
characterization of conduction barriers in sinus/atrially paced rhythm and association with intra-atrial reentrant tachycardia circuits following congenital heart disease surgery. J Cardiovasc Electrophysiol 12:17-25

[6] Akar JG, Kok LC, Haines DE, DiMarco JP, Mounsey JP (2001) Coexistence of type I atrial flutter and intra-atrial re-entrant tachycardia in patients with surgically corrected congenital heart disease. J Am Coll Cardiol 38:377-84

[7] Shigeaki Aoyagi, Koichi Arinaga, Hiroshi Tomoeda, Koji Akasu, Tomohiro Ueda (2010) Heart valve surgery in octogenarians:operative and long-term results. Heart and Vessels 25: 522-528 


\section{Figure legends}

Fig. 1 Twelve-lead electrocardiogram (ECG) showing narrow QRS tachycardia. The 12-lead ECG showed a negative $\mathrm{P}$ wave in lead $\mathrm{V}_{1}$ and positive $\mathrm{P}$ wave in lead $\mathrm{I}$ and $\mathrm{a} \mathrm{V}_{\mathrm{L}}$. QRS morphology was identical to baseline ECG.

Fig. 2 Voltage map of the right atrium and contact bipolar electrograms during coronary sinus pacing. Double potentials were noted on the posterolateral wall. This region was presumed to be the crista terminalis (blue tags A). A low voltage area was noted on the lateral wall. Split potentials (not discrete double potential) (blue tags B) and single potentials (C, D) were noted on the lateral right atrial wall. The scar and fragmented potentials area were recorded between the split potential region and the IVC (E).

SVC: superior vena cava, IVC: inferior vena cava, TV: tricuspid valve

Fig. 3 Voltage map and contact bipolar electrograms during pacing from a region close to a split potential (the star indicates the pacing site). The region of double potentials (DPs) expanded, forming a linear lesion on the lateral wall (blue tags). Abbreviations as in Fig. 2. 
Fig. 4 Activation map and contact bipolar electrograms during pacing from a region close to a split potential (the star indicates the pacing site). A single potential was noted high in the right atrium (RA) (A). DPs were noted in the lateral RA (B, D; A-A' interval $70 \mathrm{~ms}$ and 59ms, respectively). The iso-electrical line could not be seen clearly at the upper site (C; A-A' interval 29ms). Low voltage and fragmented potentials (orange tags) were noted at the lower border of the region of DPs (E).

Abbreviations as in Fig. 2.

Fig. 5 Activation map and contact bipolar elecrtograms during pacing from the opposite site of the presumed atriotomy scar identifying the same linear lesion seen in Fig. 3 (the star indicates the pacing site). The superior and inferior edges of the linear lesion were verified (A, B, C, D). Abbreviations as in Fig. 2.

Fig. 6 Activation map and contact bipolar electrograms generated during coronary sinus (CS) pacing after achievement of complete bi-directional conduction blocks between the lower end of the presumed atriotomy scar and the inferior vena cava (IVC) and across the cavotricuspid isthmus (CTI). Atrial counterclockwise activation 
descended around the tricuspid valve from the CS (A, C, D). Double potentials on the CTI are shown in panel E. Atrial activation turning posteriorly was blocked by a blockade line between the presumed atriotomy scar and the IVC (B). The red tags indicate ablation lesions. 
Fig. 1
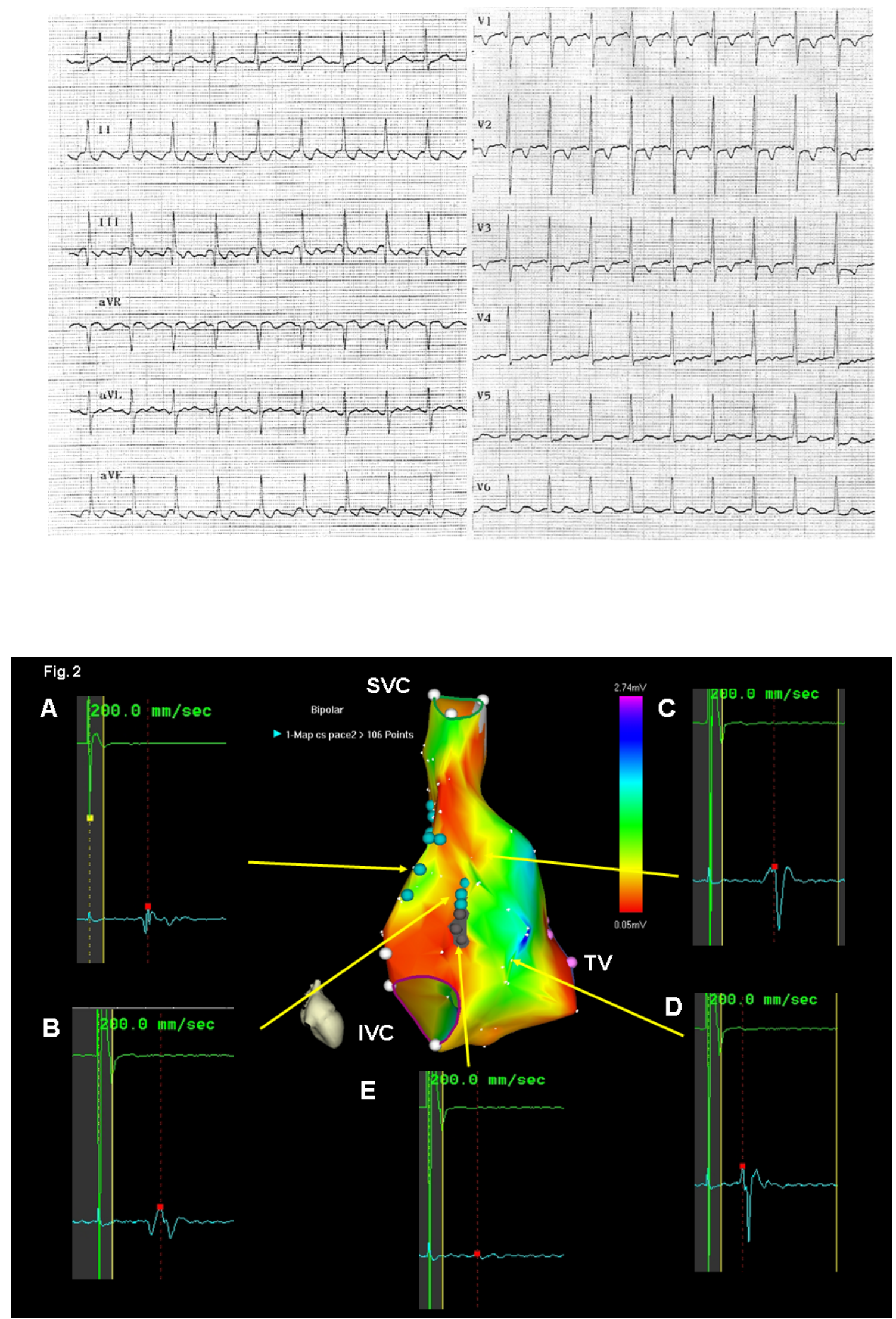

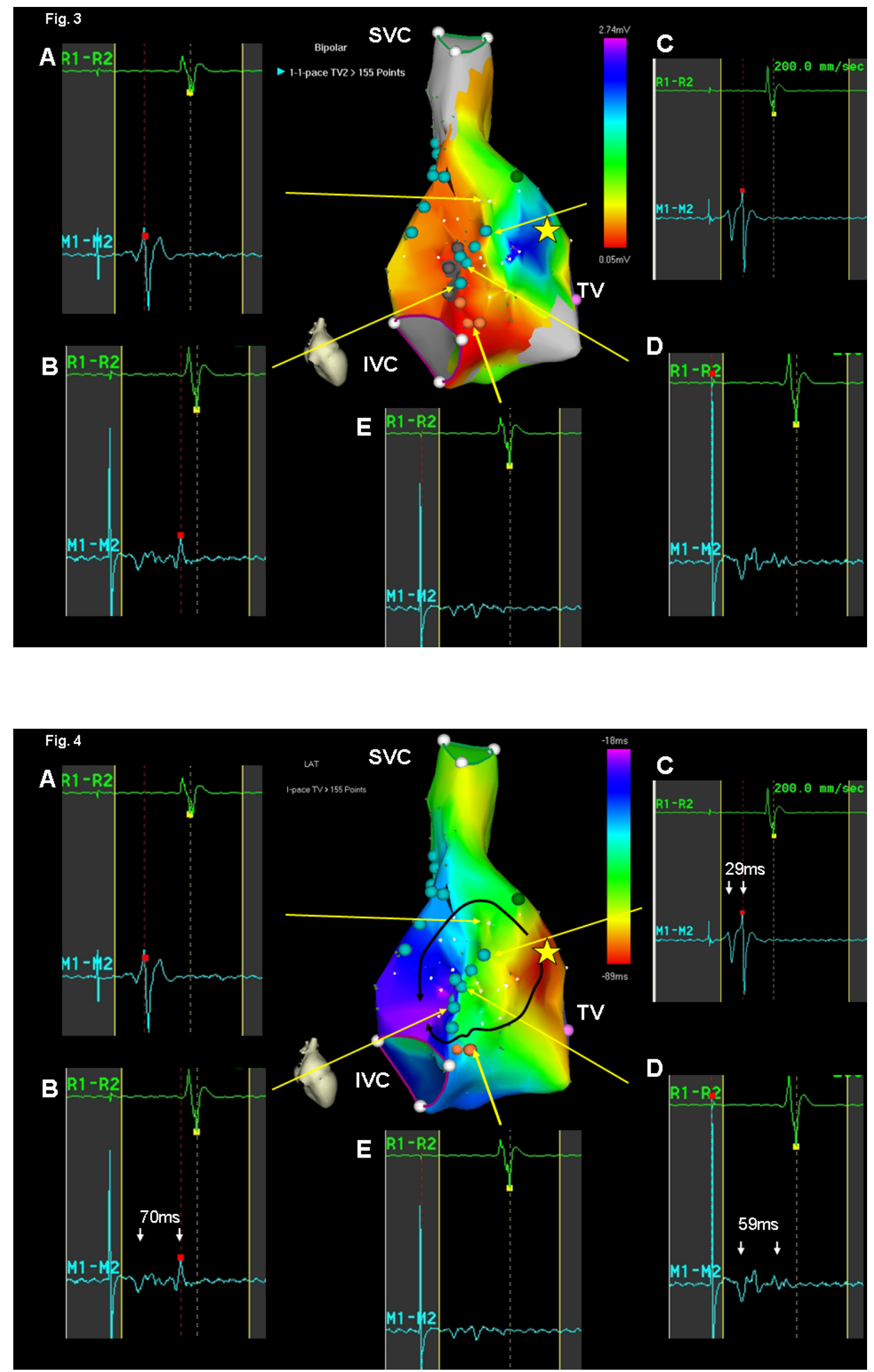

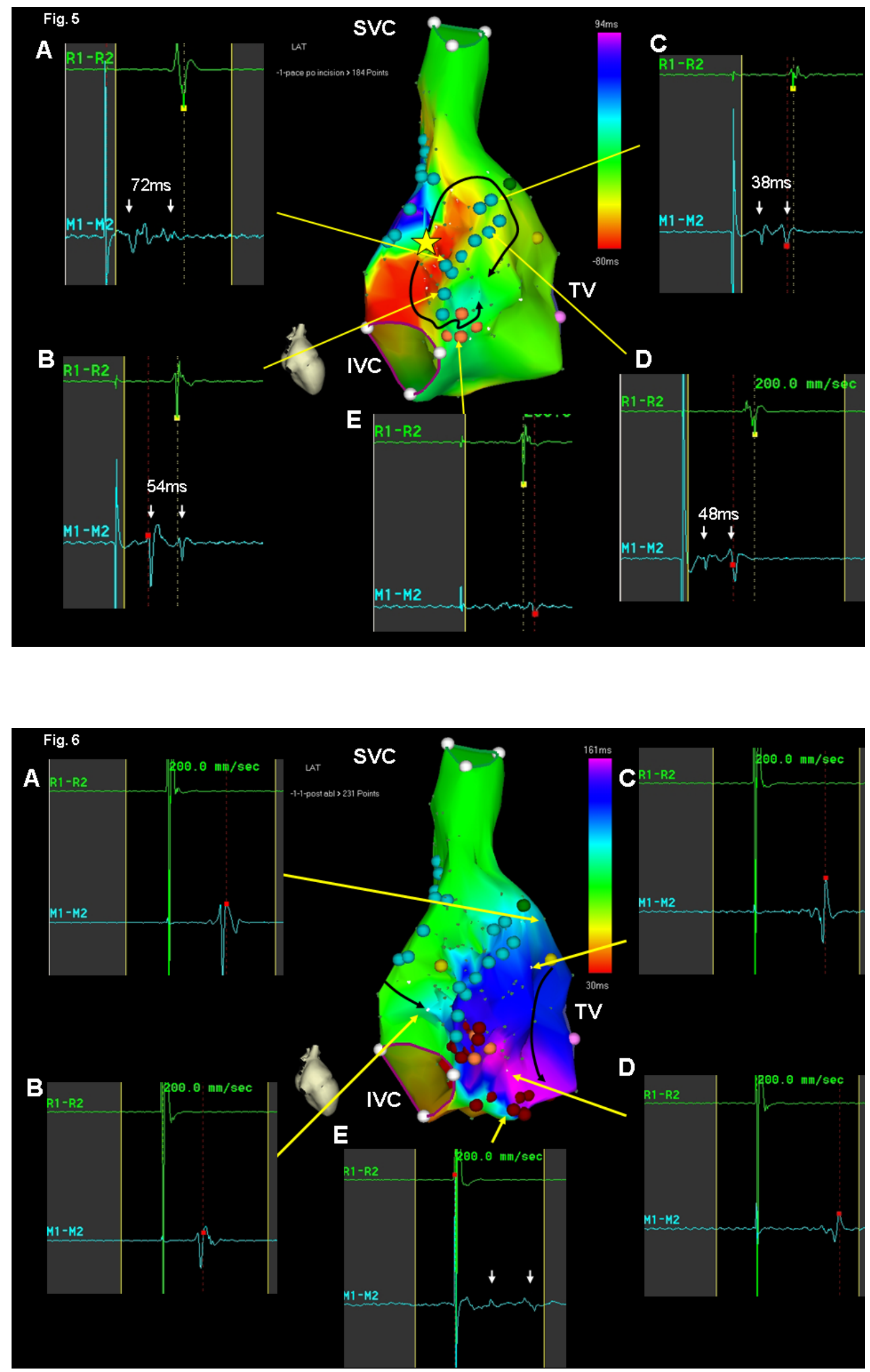